\title{
Isolation of antimicrobial resistant bacteria in upper respiratory tract infections of patients
}

\author{
Li-min Wang ${ }^{1} \cdot$ Xiao-liang Qiao ${ }^{1} \cdot$ Liang Ai $^{1} \cdot$ Jing-jing Zhai $^{1} \cdot$ Xue-xia Wang ${ }^{1}$
}

Received: 7 February 2016/Accepted: 19 July 2016/Published online: 11 August 2016

(c) The Author(s) 2016. This article is published with open access at Springerlink.com

\begin{abstract}
Haemophilus influenzae, Streptococcus pyogenes, Moraxella catarrhalis, Staphylococcus aureus, and Streptococcus pneumoniae are usual cause of upper respiratory tract infection cases. The present study aims the isolation of bacterial strains which are resistant to the commonly prescribed antibiotics. In total, 900 throat swabs were obtained from the patients suffering from upper respiratory tract infections residing in three different localities. The maximum number of isolates $(64 \%)$ were obtained from locality-1 (L-1), whereas lowest isolates were found in second locality (L-2). H. influenzae was found to be the most dominant bacterial pathogen in upper respiratory tract infections in patients with $42 \%$ of the total isolates. H. influenzae and Chlamydia pneumoniae were resistant to $\beta$-lactam antibiotics but susceptible to fluroquinolones and aminoglycosides, whereas $S$. aureus and $S$. pneumoniae were found to be highly resistant to $\beta$ lactam, aminoglycosides and fluroquinolones. S. aureus was also moderately resistant to fluroquinolones and aminoglycosides with percent resistance of 26,33 and $18 \%$, respectively. $56 \%$ S. pneumoniae isolates were resistant against erythromycin, $27 \%$ against chloramphenicol and $23 \%$ against cefuroxime. The studies revealed that $S$. aureus and $S$. pneumoniae strains were high producer of biofilms which could be one of the reasons for their high pathogenicity.
\end{abstract}

Li-min Wang

wlmwanglimin@hotmail.com

1 Department of Clinical Laboratory, Women and Infants Hospital of Zhengzhou, No. 41, Jinshui Road, Jinshui District, Zhengzhou, Henan 450012, People's Republic of China
Keywords Isolation A Antibiotic resistance · Upper respiratory tract infection $\cdot$ Biofilm

\section{Introduction}

Upper respiratory tract infections are one of the common diseases which can be found in the individuals of all age groups. Pharyngitis, nasopharyngitis, tonsillitis, otitis media and sinusitis are the major infections of upper respiratory tract. These major problems are generally caused by viruses (rhinovirus, adenovirus, parainfluenza virus, human metapneumovirus, and influenza virus. After the viral invasion, the secondary infection is caused by various types of bacteria resulting in chronic obstructive lung disease and high fever. The different types of bacteria which are involved in upper respiratory tract infections (RTIs) are Haemophilus influenzae, Streptococcus pyogenes, Moraxella catarrhalis, Staphylococcus aureus, and Streptococcus pneumonia. Although the upper respiratory tract infection cases are not complicated, the acute swelling caused by these infections may threaten airway patency resulting in asthmatic condition or cause obstruction in the passage of ingestion which further leads to significant dehydration. The RTIs are more common in developing countries in which, after diarrhea, RTIs are the second cause of death in children due to pneumonia (Ndip et al. 2009). Each year, United States reports that mortality rate of $\sim 14 \%$ (5.6 million cases) is directly associated with community-acquired pneumonia (CAP) (Karchmer 2004).

Bacteria are becoming resistant to conventional antibiotics. Ten years ago, concern centered on Gram-positive bacteria, particularly methicillin-resistant $S$. aureus and vancomycin-resistant Enterococcus spp (Kumarasamy et al. 2010). Now, however, large number of clinical 
microbiologists agree that multidrug-resistant Gram-negative bacteria pose the greatest risk to public health (Ukpai et al. 2015). The increase in resistance of Gram-negative bacteria (faster than in Gram-positive bacteria) is higher than active new/developmental antibiotics, and only a few drug development programs are insufficient to provide therapeutic cover in next 10-20 years. The main reason for increase in resistance from antibiotics in bacteria is due to the mobile genes present on the plasmids which spread and transfer through bacterial populations (Bennett 2009). The overuse and misuse of antibiotics for the treatment of respiratory tract infection has been considered one of the major reasons for the emergence of resistance of bacteria against antibiotics (Gonzales et al. 1997). Another reason for antibiotic resistance of bacteria has been considered to be the production of biofilms during quorum-sensing-regulated mechanism which releases beta-lactamase responsible for degradation of various antibiotics (Wilke et al. 2005). It has been reported that $S$. pneumoniae were found to be resistant to penicillin and erythromycin, $H$. influenzae were resistant from ampicillin, whereas S. pyogenes isolates were resistant from erythromycin (Karchmer 2004).

Nowadays, the resistance of bacteria against existing antibiotics has been at alarming stage throughout the world with high mortality rate due to acute infections by various bacteria in respiratory infections in China, so more studies are required to isolate the novel bacterial strains resistant to various antibiotics. The pool of isolated resistant bacteria will be used to develop the potential strategy so as to target the regulation of bacterial resistance mechanisms to enhance the potency of available drugs, restore the efficacy of available drugs and develop the new range of antibiotics specific to new mechanism of drug resistance (Lister et al. 2009). In the present study, attempts were made to isolate the bacterial species from the infected persons of different age groups residing in different localities in China which were resistant against commonly prescribed antibiotics in China.

\section{Materials and methods}

\section{Materials}

Sterilized swabs were purchased from Fisher scientific (UK); Blood agar and eosin methylene blue agar were purchased from Sigma Aldrich (USA); for biochemical tests, API 20E kit was purchased from Biomerieux (France) and Mueller-Hinton plates were purchased from Biotech (England). All other laboratory reagents were purchased from Merck-Millipore Chemicals.

\section{Experimental designs and subjects}

The studies were conducted in Guangdong Province, China. The province is thickly populated and is about $1902 \mathrm{~m}$ above the sea level. Average daily temperature in Guangzhou in January and July are 18 and $33{ }^{\circ} \mathrm{C}$, respectively, with high humidity. The favorable temperature and relatively high humidity are mainly responsible for the respiratory tract infections.

The study was conducted on three different localities and three different age groups. The first group was having subjects with age ranging 5-25 years, which were mainly students studying in schools and colleges. The second group was 26-45 years age group which consists of working-class people and the third age group was ranging 46-65-year-old people. Totally, 900 subjects were selected (300 persons in each locality), and each locality includes 155 males and 145 female subjects. The subjects were informed prior about the aim of study, and their verbal consent was solicited. All the subjects were assured regarding privacy of disease and data. The three different localities were designated as L-1, L-2 and L-3. The different age groups, i.e., 5-25 years, 26-45 years and 46-65 year were designated as $\mathrm{X}, \mathrm{Y}$ and $\mathrm{Z}$, respectively. All the subjects included in the study were having symptoms of sore throat, cough, running nose, and the study was conducted between March 2014 and October 2014 .

\section{Isolation of bacterial strains}

Three hundred throat swabs from individuals of different age groups were taken in the sterilized plastic sample bags for the study. The samples were collected between 6 months (March 2014-August 2014) which included both dry and wet seasons which is favorable for the growth and invasion of various microorganisms.

The swabs were aseptically inoculated on the Petri plates containing blood agar media, and the plates were incubated at $37^{\circ} \mathrm{C}$ in incubator for $24 \mathrm{~h}$. After incubation, the morphological as well as microscopic examination of the colonies was done. The colonies obtained on the petri plates were subcultured on solid media several times to get the pure culture of the microorganisms. When the pure cultures were obtained, the microorganisms were subcultured on the solid media in slants and preserved at $4{ }^{\circ} \mathrm{C}$ for further investigations. Microorganisms in pure culture were identified on the basis of their morphological characteristics on selective and differential media. API 20E kits were used for biochemical testing to confirm the identity of microorganisms. 


\section{Antibiotic susceptibility test}

Antibiotic susceptibility test using microbroth dilution method was used to investigate the antibiotic susceptibility/ resistance of isolates (Hendriksen 2003; Tilton et al. 1973). In brief, the test was carried out in a 96-well microtiter plate by distributing $240 \mu \mathrm{L}$ of sterile media and $30 \mu \mathrm{L}$ of antibiotic stock $\left(5 \mathrm{mg} \mathrm{mL}^{-1}\right)$ giving concentration of $500 \mu \mathrm{g} \mathrm{mL}^{-1} ; 150 \mu \mathrm{L}$ from first well was transferred to the next and was diluted with sterile medial with factor of 2 . Dilution up to 10 wells were giving the range of 500-0.197 $\mu \mathrm{g} \mathrm{mL}^{-1}$, and appropriate blanks (to have a check on sterility) and controls (to see the growth-favoring ability of media) were also taken. To each well (except blank), $10 \mu \mathrm{L}$ of isolated and identified bacterial inocula $\left(10^{7}\right.$ cells $\left.\mathrm{mL}^{-1}\right)$ were added, and plate was incubated in rotary shaker incubator $150 \mathrm{rpm}, 30^{\circ} \mathrm{C}$ for $36 \mathrm{~h}$. The qualitative observation of turbidity was carried out with naked eyes under light and dark background and compared with clear blank well and hazy control well to make a decision of growth. The minimum inhibitory concentration (MIC) of particular antibiotic against particular organism was defined as lowest concentration where no viability of bacteria was observed in the wells of 96-microwell plates after incubation of $36 \mathrm{~h}$.

\section{Detection of biofilm formation}

Most of the antibiotic-resistant bacteria form biofilms during their growth and metabolism which are responsible for their resistance against many antibiotics. Two different biofilm formation analysis tests were performed. First test was based on the colorimetric detection of crystal violetstained bacteria which is also known as tissue culture plate test (TCP) (Stepanovic et al. 2007). Another method for detection of biofilm was used which was based on the Congo red dye test (Freeman et al. 1989). In brief, the stock strain was transferred to Mueller-Hinton agar and was incubated for $24 \mathrm{~h}, 37^{\circ} \mathrm{C}$. Purified strain culture was resuspended in phosphate buffer $\mathrm{pH} 7.050 \mathrm{mM}$ and diluted appropriately to get turbidity equivalent to $0.5 \mathrm{McFarland}$ standard, i.e., $10^{8} \mathrm{CFU} / \mathrm{mL}$. Sterile tryptic soy broth supplemented with $2.5 \%$ glucose was taken for inoculation of isolated bacteria and were incubated for $24 \pm 0.5 \mathrm{~h}$ at $37^{\circ} \mathrm{C}$ with no shaking. Gentle washing was done with $300 \mu \mathrm{L}$ phosphate buffer $(\mathrm{pH} 7.0,50 \mathrm{mM})$ five times. Biofilm fixation was done through $150 \mu \mathrm{L}$ methanol exposure for $15 \mathrm{~min}$. The wall-fixed biofilm-containing bacteria was stained with $200 \mu \mathrm{L}$ of $2 \%$ crystal violet. After 15 min exposure, rest of the stain was removed and washed with buffer till no dye appeared in the wash. The well plate is dried at room temperature; then, the dried,stained,wall-fixed biofilm is solubilized by $95 \%$ ethanol for $30 \mathrm{~min}$, and OD was measured at 570 .

\section{Results}

\section{Bacterial isolates}

The dominated species of pathogenic bacteria in three different localities of Guangdong Province of China has been presented in Table 1. From the various inoculated samples, $59 \%$ of the samples examined were positive for infectious microorganisms. The four dominated species of pathogenic bacteria were identified as $H$. influenzae, $S$. aureus, S. pneumoniae and Chlamydia pneumonia. Each isolate/strain was given a code with respect to the species, e.g., H.I.1 for first isolate/strain of $H$. influenza and similarly for other species. Among four bacterial species, the highest number $(23 \%)$ of isolates of $H$. influenzae were obtained, whereas lowest number $(8 \%)$ were obtained of $C$. pneumoniae, whereas $S$. aureus and $S$. pneumoniae contributed 15 and $13 \%$ of total isolates, respectively. It was observed that the maximum $(64 \%)$ number of isolates were obtained from locality-1 (L-1), whereas lowest isolates were found in the second locality (L-2).

It was observed that among three different age groups, the maximum isolates $(51.03 \%)$ were obtained from the age group of 5-25 years among which $42 \%$ of isolates were $H$. influenzae as shown in Table 2.

\section{Antibiotic susceptibility test}

Microbroth dilution method was used to test the susceptibility of isolated microorganisms against different antibiotics such as ampicillin, amoxyclav, ciprofloxacin, chloramphenicol, erythromycin, cefuroxime and gentamicin ranging from $(0.02-64 \mu \mathrm{g} / \mathrm{mL})$, and criteria for suggesting whether the isolate is susceptible or resistant toward specific antibiotic have been given in Table 3. It was observed that the $H$. influenzae was highly resistant to ampicillin (43\% resistant strains) and erythromycin (41\% resistant strains), whereas it was least resistant to ciprofloxacin (9\% resistant strains) and gentamicin (12\% resistant strains) (Table 4).

Staphylococcus aureus was resistant to $\beta$-lactam antibiotics such as ampicillin, amoxyclav as well as marcolide antibiotics such as erythromycin with percent resistance of 65,46 and $54 \%$, respectively (Table 4). S. aureus was also moderately resistant to fluroquinolone antibiotics such as ciprofloxacin, chloramphenicol and aminoglycosides antibiotics such as gentamicin with percent resistance of 26,33 and $18 \%$, respectively. 
Table 1 Different bacterial isolates in three different localities

\begin{tabular}{llll}
\hline Bacterial species & Locality & $\begin{array}{l}\text { Number of } \\
\text { isolates }\end{array}$ & $\begin{array}{l}\text { Percentage (\%) } \\
\text { of isolates }\end{array}$ \\
\hline Haemophilus influenzae & L-1 & 78 & 26 \\
& L-2 & 45 & 15 \\
& L-3 & 86 & 28.66 \\
Staphylococcus aureus & L-1 & 41 & 13.66 \\
& L-2 & 55 & 18.33 \\
& L-3 & 38 & 12.66 \\
Streptococcus pneumoniae & L-1 & 46 & 15.33 \\
& L-2 & 32 & 10.66 \\
& L-3 & 40 & 13.33 \\
Chlamydia pneumoniae & L-1 & 28 & 9.33 \\
& L-2 & 22 & 7.33 \\
& L-3 & 20 & 6.66 \\
Total & & 531 & 59 \\
\hline
\end{tabular}

In this study, S. pneumoniae was observed to be $56 \%$ resistant against erythromycin, $27 \%$ against chloramphenicol and $23 \%$ against cefuroxime (Table 4). It was observed that comparative to other isolates, $S$. pneumoniae was even 42 and $21 \%$ resistant against ciprofloxacin and gentamicin which were found to be highly sensitive antibiotics against other isolates. C. pneumoniae was observed to be highly resistant against chloramphenicol and erythromycin compared to other antibiotics; it was having 57 and $63 \%$ resistance against chloramphenicol and erythromycin, respectively (Table 4).

\section{Biofilm production test}

Many pathogenic microorganisms form a slimy layer during invasion and infections which is basically termed as biofilm. All isolates were studied for biofilm production using tissue culture plate (TCP) test and Congo red dye test. Purple-bluish color was observed in the tissue culture plate wells when stained with gram stain. The organisms which produced dark purple-bluish color in tissue culture wells were considered as strong biofilm- producing strains, the bacteria with light purple-bluish color stained in wells were considered as moderate biofilm-producing strains, whereas the bacteria which were not stained in the wells were considered as no biofilmproducing strains (Fig. 1a).

It was observed that among four major isolates, $S$. aureus and $S$. pneumoniae were found positive for biofilm production. S. pneumoniae and $S$. aureus strains were observed to produce strong biofilms, whereas few strains of $H$. influenzae and $C$. pneumoniae were producing moderate biofilms with light purple color staining in the micro-titre plate wells and maximum strains were found to be negative for biofilm production with no purple color staining in the tissue culture plate wells. The strong and moderate biofilm-producing strains were further tested by Congo red dye test method in Petri plates. It was observed that the strong biofilm-producing S.A.11 strain of $S$. aureus and S.P.5 strain of S. pneumoniae were observed to produce dark black-colored colonies in congo red plate indicating strong biofilms production, whereas C.P.24 strain of C. pneumoniae and H.I.98 strain of H. influenzae which have shown moderate biofilm production in TCP test were able to produce black-colored colonies in congo red dye plate indicating no biofilm production (Fig. 1b). The SEM images of $S$. aureus and S. pneumoniae in Fig. 1c clearly showed the biofilm production in these strains.

\section{Discussion}

From the results in Table 1, it was observed that the maximum (64\%) number of isolates was obtained from locality-1 (L-1), whereas lowest isolates were found in second locality (L-2). The possible reason for maximum isolates from L-1 could be the unhygienic living standards as well as damp and humid environment which were the favorable conditions for the growth and development of various pathogens. Moreover, the locality was thickly populated which had also increased the risk of communicable infections.

Table 2 Percentage of bacterial isolates found in three different age groups

\begin{tabular}{|c|c|c|c|c|c|c|c|c|}
\hline \multirow[t]{3}{*}{ Bacterial species } & \multicolumn{8}{|c|}{ Age groups (years) } \\
\hline & \multicolumn{2}{|l|}{$5-25$} & \multicolumn{2}{|l|}{$26-45$} & \multicolumn{2}{|l|}{$46-65$} & \multicolumn{2}{|l|}{ Total } \\
\hline & Isolates (no.) & $\%$ & Isolates (no.) & $\%$ & Isolates (no.) & $\%$ & Isolates (no.) & $\%$ \\
\hline Haemophilus influenzae & 126 & 42 & 23 & 7.6 & 60 & 20 & 209 & 23.22 \\
\hline Staphylococcus aureus & 63 & 21 & 30 & 10 & 41 & 13.66 & 134 & 14.88 \\
\hline Streptococcus pneumoniae & 50 & 16.66 & 38 & 12.66 & 40 & 13.33 & 118 & 13.11 \\
\hline Chlamydia pneumoniae & 32 & 10.66 & 18 & 6 & 20 & 6.6 & 70 & 7.77 \\
\hline Total & 271 & 51.03 & 109 & 20.52 & 161 & 30.32 & 531 & 59 \\
\hline
\end{tabular}


Table 3 Interpretation of susceptible or resistant bacteria and the accepted range of MIC for the E. coli ATCC 25922 reference strain

\begin{tabular}{llll}
\hline Antibiotic & $\begin{array}{l}\text { MIC interpretive criteria } \\
(\mu \mathrm{g} / \mathrm{mL})\end{array}$ & $\begin{array}{l}\text { E. coli ATCC } \\
25922 . \mathrm{MIC} \\
(\mu \mathrm{g} / \mathrm{mL})\end{array}$ \\
\cline { 2 - 3 } & $\begin{array}{l}\text { Susceptible } \\
(\mathrm{S})\end{array}$ & $\begin{array}{l}\text { Resistance } \\
(\mathrm{R})\end{array}$ & \\
\hline Ampicillin (AMP) & $\leq 4$ & $\geq 16$ & 4 \\
Amoxyclav (AMC) & $\leq 1$ & $\geq 4$ & 1 \\
Amoxicillin (AMO) & $\leq 64$ & $\geq 128$ & 64 \\
Ciprofloxacin (CIP) & $\leq 0.08$ & $\geq 0.32$ & 0.08 \\
Chloramphenicol (CHL) & $\leq 4$ & $\geq 16$ & 4 \\
Erythromycin (ERY) & $\leq 0.2$ & $\geq 0.8$ & 0.2 \\
Cefuroxime (CEF) & $\leq 4$ & $\geq 16$ & 4 \\
Gentamicin (GEN) & $\leq 0.2$ & $\geq 0.8$ & 0.2 \\
\hline
\end{tabular}

Table 2 shows bacterial isolates found in three different age groups, and depicts that $H$. influenzae was the most dominant during secondary respiratory tract infections in children. This age group included mostly the school/college going children. Many students were found to be underweight, malnourished and debilitated due to which they were having lower immunity and more vulnerable to the respiratory infections. The minimum isolates (20.52\%) were obtained from the age group of 26-45 years among which the $12.66 \%$ isolates were $S$. pneumoniae as this age group was basically composed of the healthy adults working in hygienic conditions and found to have good immunity against infections. In the age group of 46-65 years which was mostly composed of old-age persons, $30.32 \%$ of isolates were obtained with $20 \%$ of $H$. influenzae.

Antibiotic susceptibility studies showed $H$. influenzae to be highly resistant to ampicillin and erythromycin, however, it was least resistant to ciprofloxacin and gentamicin. It has been reported that in $H$. influenzae, the resistance from ampicillin and amoxicillin was mediated by TEM-1 $\beta$-lactamase enzyme (Rennie and Ibrahim 2005). In previous studies, chloramphenicol was considered as the best antibiotic against $H$. influenzae, but few studies in north India revealed no resistance from this drug (Puri et al.
1999). Ampicillin inhibits the cell wall synthesis of bacteria by binding to penicillin-binding proteins (PBPs) so, maybe due to mutations or environmental changes in bacteria, ampicillin was not able to bind with the PBPs resulting in bacterial resistance to ampicillin (Sharma et al. 2013). Also, erythromycin was not able to inhibit the growth of $H$. influenzae even at higher concentrations which might be due to some protective mechanism that it developed against erythromycin. Few studies had also reported $H$. influenzae resistance toward ciprofloxacin due to mutations in the quinolone resistance-determining regions (QRDRs) of gyrA and parC (Brenwald et al. 2003). Ciprofloxacin inhibits the bacterial replication, transcription and repair by inactivating topoisomerase II (DNA gyrase) and topoisomerase IV enzymes. But in this study, $H$. influenzae showed no resistance against ciprofloxacin. Along with ciprofloxacin, gentamicin was also found to be effective against $H$. influenzae strains due to its irreversible binding to $30 \mathrm{~S}$ units of bacterial ribosomes which inhibits its protein synthesis. Ciprofloxacin and gentamicin both were reported to be susceptible for $H$. influenzae in previous studies (Zanchi et al. 1994).

On the other hand, $S$. aureus was resistant to $\beta$-lactam antibiotics as well as marcolide antibiotics. Although $S$. aureus has broad-spectrum resistance against different class of antibiotics such as $\beta$-lactams, glycopeptides, quinolones, aminoglycosides, oxazolidinones, quinupristindalfopristin due to presence of various resistance genes (Lowy 2003), in another study, S. aureus was found to be resistant against methicillin antibiotics due to presence of mec and ccr genes (Chambers and Deleo 2010); but in this study, ciprofloxacin, chloramphenicol and gentamicin were found to be highly susceptible for $S$. aureus with low resistance. Due to the horizontal gene transfer of complex genetic array of mec element or vanA operon, it became resistant to different types of antibiotics (Pantosti et al. 2007). Cefuroxime was having moderate effect on S. aureus with $61 \%$ sensitivity.

S. pneumoniae is a major causative agent in respiratory tract infections in humans, and they were once highly penicillin-susceptible microorganisms, but recently, they

Table 4 Antibiotic resistance test of different bacterial isolates with different antibiotics

\begin{tabular}{|c|c|c|c|c|c|c|c|}
\hline \multirow[t]{2}{*}{ Isolates and number tested } & \multicolumn{7}{|c|}{ No. of isolates resistant to antibiotics } \\
\hline & Ampicillin & Amoxyclav & Ciprofloxacin & Chloramphenicol & Erythromycin & Cefuroxime & Gentamicin \\
\hline Haemophilus influenzae (209) & $90(43 \%)$ & $48(23 \%)$ & $25(12 \%)$ & $56(27 \%)$ & $85(41 \%)$ & $32(15 \%)$ & $18(9 \%)$ \\
\hline Staphylococcus aureus (134) & $87(65 \%)$ & $62(46 \%)$ & $35(26 \%)$ & $44(33 \%)$ & $72(54 \%)$ & $52(39 \%)$ & $24(18 \%)$ \\
\hline Streptococcus pneumoniae (118) & $63(53 \%)$ & $45(38 \%)$ & $50(42 \%)$ & $32(27 \%)$ & $66(56 \%)$ & $27(23 \%)$ & $25(21 \%)$ \\
\hline Chlamydia pneumoniae (70) & $40(57 \%)$ & $16(23 \%)$ & $12(17 \%)$ & $38(54 \%)$ & $44(63 \%)$ & $12(17 \%)$ & $14(20 \%)$ \\
\hline Total & $280(53 \%)$ & $171(32 \%)$ & $122(23 \%)$ & $170(32 \%)$ & $267(50 \%)$ & $123(23 \%)$ & $81(15 \%)$ \\
\hline
\end{tabular}




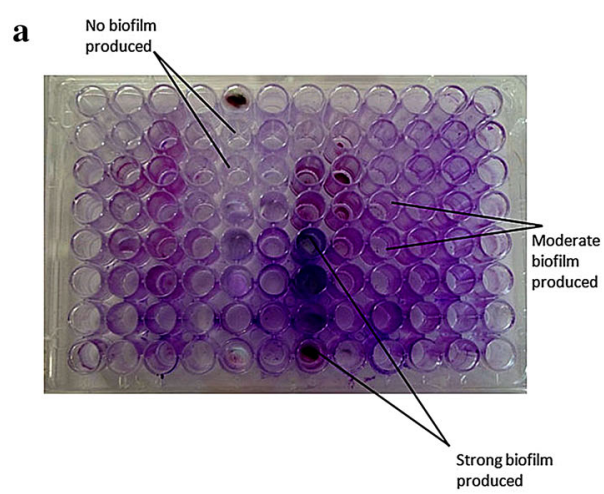

b

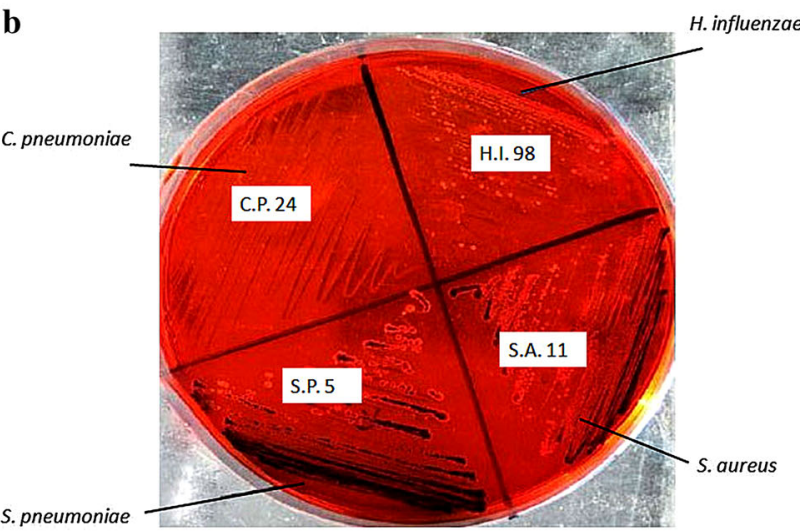

c
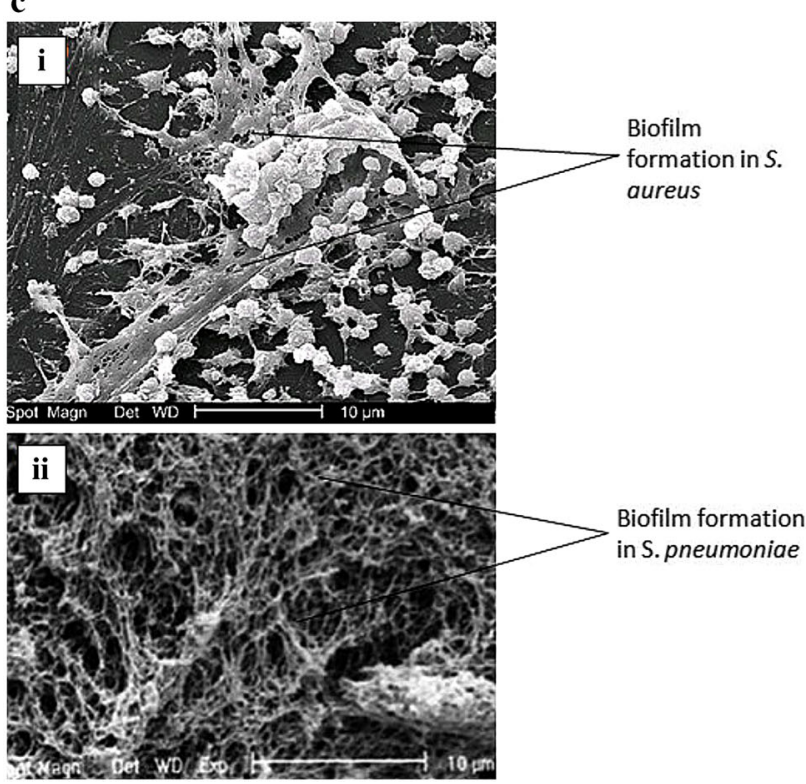

Fig. 1 Tissue culture plate (TCP) test for biofilm production in bacterial isolates (a), Congo red dye test for biofilm production in isolates (b) and SEM images of biofilm produced in (i) S. aureus and (ii) S. pneumoniae (c)

were found to be multidrug-resistant (Tomasz 1997). $S$. pneumoniae has been found to be responsible for estimated one million deaths each year in children below 5 years (Obaro et al. 1996). Susceptibility of $S$. pneumoniae was studied in Canada, and it was observed that ciprofloxacin was having poor potency against S. pneumoniae (Patel et al. 2011). After 10 years of use in United States, the susceptibility of ciprofloxacin was examined with $S$. pneumoniae, and the MIC was observed to be increased due to bacterial resistance (Sahm et al. 2000).

C. pneumoniae was observed to be highly resistant against chloramphenicol and erythromycin in this study. In previous studies, it was observed that $C$. pneumoniae is susceptible to erythromycin, azithromycin, roxithromycin, clarithromycin, doxycycline, ofloxacin, and rifampin, and their combination is more lethal (Freidank et al. 1999) which was in agreement with our study.

\section{Conclusion}

The study revealed that various pathogenic bacteria in respiratory tract infection had become resistant to wide range of commonly used antibiotics. H. influenzae was the major causative agent for respiratory infections found in all age groups as its maximum number of isolates was obtained in the present study. Although considerable percentage of all isolates were observed to be resistant to various antibiotics, S. aureus and $S$. pneumoniae were found to be highly resistant to strong antibiotics like ciprofloxacin and gentamicin which might be due to the presence of resistant genetic elements present in these strains. The emergence of highly resistant bacteria against the commonly prescribed antibiotics is at alarming level; therefore, novel antibiotics should be introduced for severe respiratory tract infections. Moreover, different antibiotics in combination can be helpful against various resistant pathogenic strains.

In few studies, $H$. influenzae has been reported to produce strong biofilm (Galli et al. 2007; Mizrahi et al. 2014), but in the present study, strong biofilm production in $H$. influenzae strains was not observed. One of the possible reasons for biofilm production in various bacteria is quorum sensing. Quorum sensing in S. aureus was found to be responsible for the production of biofilms during various infections, and gene regulator $a g r$ was found to play important role in its biofilm production (Yarwood et al. 2004). The production of biofilms in bacteria has been reported to enhance the bacterial resistance against antibiotic, for example, production of biofilms in S. aureus was responsible for its resistance against methicillin (McCarthy et al. 2015). In another study, $60.47 \%$ of $S$. aureus were found to be biofilm producers when tested with crystal violet test method, while $13.95 \%$ of strains were found to be biofilm producer when tested with congo red agar (CRA) test method (Elkhatib et al. 2014) which showed high variability in these two methods which is in 
agreement with our study. In previous studies, S. pneumoniae was also observed to be good producer of biofilm during respiratory tract infections (Moscoso et al. 2006) which is in agreement with this study.

\section{Compliance with ethical standards}

Conflict of interest $\mathrm{We}$, the authors, declare that we have no conflict of interest in the publication.

Open Access This article is distributed under the terms of the Creative Commons Attribution 4.0 International License (http:// creativecommons.org/licenses/by/4.0/), which permits unrestricted use, distribution, and reproduction in any medium, provided you give appropriate credit to the original author(s) and the source, provide a link to the Creative Commons license, and indicate if changes were made.

\section{References}

Bennett PM (2009) Plasmid encoded antibiotic resistance: acquisition and transfer of antibiotic resistance genes in bacteria. $\mathrm{Br} \mathrm{J}$ Pharmacol 153:S347-S357

Brenwald NP, Andrews JM, Jevons G, Wise R (2003) Detection of ciprofloxacin resistance in Haemophilus influenzae using nalidixic acid and BSAC methodology. J Antimicrob Chemother 51:1311-1312

Chambers HF, Deleo FR (2010) Waves of resistance: Staphylococcus aureus in the antibiotic ERA. Nat Rev Microbiol 7:629-641

Elkhatib WF, Khairalla AS, Ashour HM (2014) Evaluation of different microtiter plate-based methods for the quantitative assessment of Staphylococcus aureus biofilms. Future Microbiol 9:725-735

Freeman DJ, Falkiner FR, Keane CT (1989) New method for detecting slime production by coagulase negative staphylococci. J Clin Pathol 42:872-874

Freidank HM, Losch P, Vogele H, Wiedmann-Al-Ahmad M (1999) In vitro susceptibilities of Chlamydia pneumoniae isolates from German patients and synergistic activity of antibiotic combinations. Antimicrob Agents Chemother 43:1808-1810

Galli J, Calo L, Ardito F, Imperiali M, Bassotti E, Fadda G, Paludetti G (2007) Biofilm formation by Haemophilus influenzae isolated from adeno-tonsil tissue samples, and its role in recurrent adenotonsillitis. Acta Otorhinolaryngol Ital 27:134-138

Gonzales R, Steiner J, Sande M (1997) Antibiotic prescribing for adults with colds, upper respiratory tract infections, and bronchitis by ambulatory care physicians. JAMA 278:901-904

Hendriksen RS (2003) Global Salm-Surv. A global Salmonella surveillance and laboratory support project of the World Health Organization: 1-11. www.antimicrobialresistance.dk/data/ images/salmonella4_pdf.pdf

Karchmer AW (2004) Increased antibiotic resistance in respiratory tract pathogens: PROTEKT US - an update. Clin Infect Dis Off Publ Infect Dis Soc Am 39:S142-S150

Kumarasamy KK, Toleman MA, Walsh TR, Bagaria J, Butt F, Balakrishnan R, Chaudhary U, Doumith M, Giske CG, Irfan S (2010) Emergence of a new antibiotic resistance mechanism in India, Pakistan, and the UK: a molecular, biological, and epidemiological study. Lancet Infect Dis 10:597-602

Lowy F (2003) Antimicrobial resistance: the example of Staphylococcus aureus. J Clin Investig 111:1265-1273
McCarthy H, Rudkin JK, Black NS, Gallagher L, O'Neill E, O'Gara JP (2015) Methicillin resistance and the biofilm phenotype in Staphylococcus aureus. Front Cell Infect Microbiol 5:1-9

Mizrahi A, Cohen R, Varon E, Bonacorsi S, Bechet S, Poyart C, Levy C, Raymond J (2014) Non typable-Haemophilus influenzae biofilm formation and acute otitis media. BMC Infect Dis 14:1-10

Moscoso M, García E, López R (2006) Biofilm formation by Streptococcus pneumoniae: role of choline, extracellular DNA, and capsular polysaccharide in microbial accretion. J Bacteriol 188:7785-7795

Ndip RN, Ntiege EA, Ndip LM, Akoachere JFT, Akenji TN (2009) Antimicrobial resistance of bacterial agents of the upper respiratory tract of school children in Buea, Cameroon. J Health Popul Nutr 26:397-404

Obaro SK, Monteil MA, Henderson DC (1996) The pneumococcal problem. Br Med J 312:1521

Pantosti A, Sanchini A, Monaco M (2007) Mechanisms of antibiotic resistance in Staphylococcus aureus. Future Microbiol 2:323-334

Patel SN, McGeer A, Melano R, Tyrrell GJ, Green K, Pillai DR, Low DE (2011) Susceptibility of Streptococcus pneumoniae to fluoroquinolones in Canada. Antimicrob Agents Chemother 55:3703-3708

Puri J, Talwar V, Juneja M, Agarwal KN, Gupta HC (1999) Prevalence of anti-microbial resistance among respiratory isolates of Haemophilus influenzae. Indian Pediatr 36:1029-1031

Rennie RP, Ibrahim KH (2005) Antimicrobial resistance in Haemophilus influenzae: how can we prevent the inevitable? Commentary on antimicrobial resistance in $H$. influenzae based on data from the targeted surveillance program. Clin Infect Dis 41:S234-S238

Sahm DF, Peterson DE, Critchley IA, Thornsberry C (2000) Analysis of ciprofloxacin activity against Streptococcus pneumoniae after 10 years of use in the United States. Antimicrob Agents Chemother 44:2521-2524

Sharma SK, Singh L, Singh S (2013) Comparative study between penicillin and ampicillin. Sch. J Appl Med Sci 1:291-294

Stepanovic S, Vukovic D, Hola V, Di Bonaventura G, Djukic S, Cirkovic I, Ruzicka F (2007) Quantification of biofilm in microtiter plates: overview of testing conditions and practical recommendations for assessment of biofilm production by staphylococci. APMIS 115:891-899

Tilton RC, Lieberman L, Gerlach EH (1973) Microdilution antibiotic susceptibility test: examination of certain variables. Appl Microbiol 26:658-665

Tomasz A (1997) Antibiotic resistance in Streptococcus pneumoniae. Clin Infect Dis 24:S85-S88

Ukpai AE, Ngozi ME, Adam M (2015) Enzymatic inactivation of penicillins: an emerging threat to global public health. Int $\mathrm{J}$ Pharm Sci Res 6:3276-3284

Wilke MS, Lovering AL, Strynadka NCJ (2005) $\beta$-Lactam antibiotic resistance: a current structural perspective. Curr Opin Microbiol 8:525-533

Yarwood JM, Bartels DJ, Volper EM, Greenberg EP (2004) Quorum sensing in Staphylococcus aureus biofilms. J Bacteriol 186:1838-1850

Zanchi A, Mencarelli M, Sansoni A, Rossolini A, Cellesi C (1994) Antibiotic susceptibility of 206 from children in central Italy Haemophilus influenzae isolates collected. Eur J Epidemiol 10:699-702 